Agricultural Economics and Social Science
Available online at http://zjar.journals.ekb.eg

\title{
أثر انخفاض سعر الفائدة على معدل البطالة في اليابان
}

هاني محمد سليم هواري 1* ـ أحمد فوزي حامد2

1- قسم در اسات وبحوث العلوم السياسية والاقتصادية ـ معهد الدراسات والبحوث الآسيوية ـ جامعة الزقازيق ـ مصر

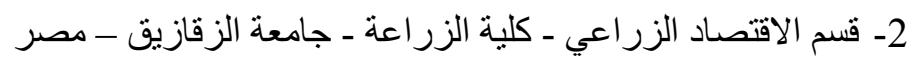

\section{Received: 7/3/2021 ; Accepted: 21/3/2021}

الملخص: سعر الفائدة يعتبر احد اهم أدوات السياسة النقدية والتي حازت على اهتمامات كبيرة في الفكر الاقتصادي عبر

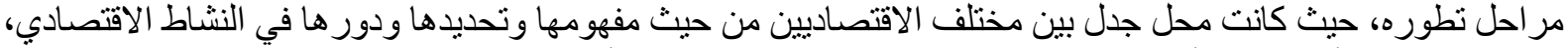

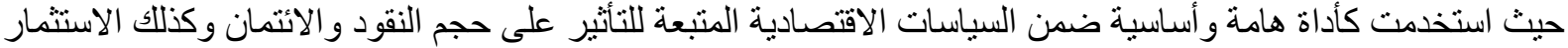

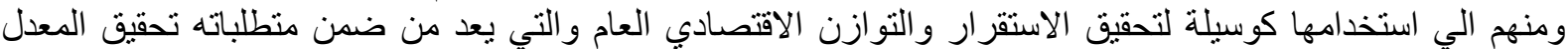

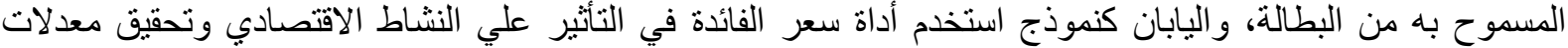

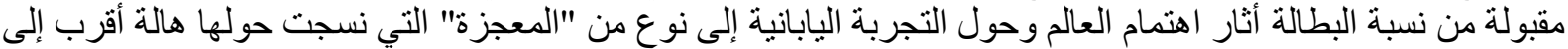

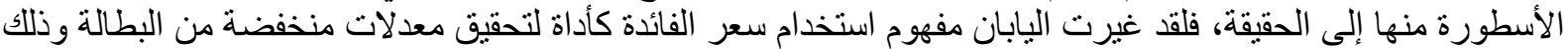

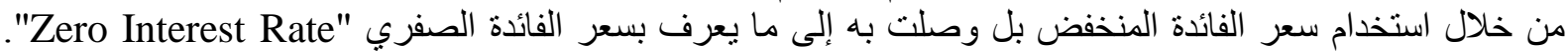

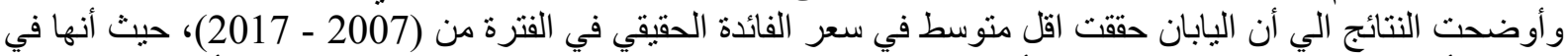

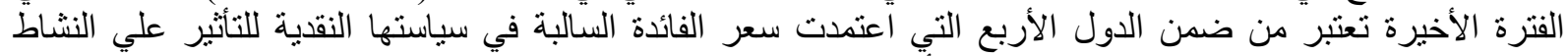

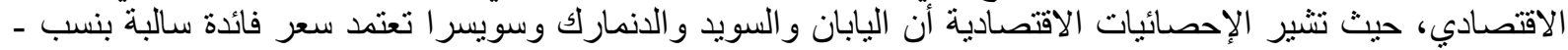

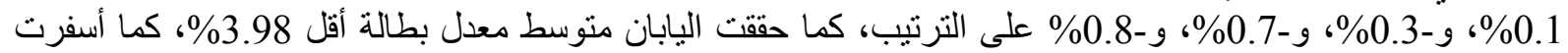
نتائج التحليل القياسي للبيانات أن سعر الفائدة يتناسب طردي مع مع معدل البطالة في اليابان. الكلمات الإسترشادية: اليابان، سعر الفائدة، السياسة النقدية، معدل البطالة.

ركود ذللك المعروض داخل حيز الادخار ومنه الي الإني

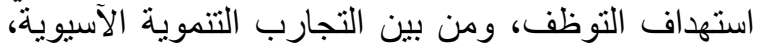

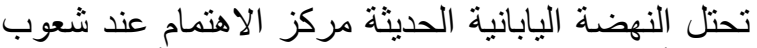

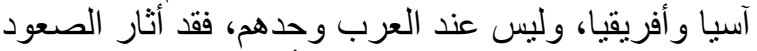

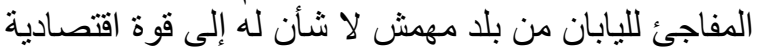

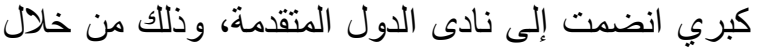

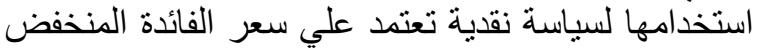

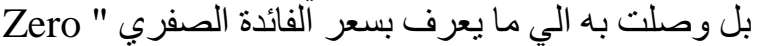

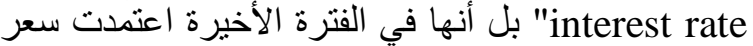
الفائدة السالبة في سياستها النقدية.

\section{المشكلة البحثية}

تتمثل مشكلة البحث في الإجابة على التساؤل التالي: هل انخفاض سعر الفائدة يؤثر على معدل البطالة في اليابان؟

$$
\text { هدف الدراسة هن }
$$

يستهدف البحث التعرف على سياسة سعر الفائدة في اليابان وقياس أثرها على تخفيض معدلات البطالة وذلتك من خلال تحليل بيانات فترة الدر اسة (1981-2017).

\section{المقدمة والمشكلة البحثية}

بدأ تحديد سعر الفائدة كظاهرة اقتصادية حقيقية في ورئية نهاية فترة العصور الوسطي في أوروبا وبداية عصر الئهر النهضة وظهور الر أسمالية التجارية، نتيجة تغير التئية الظروف

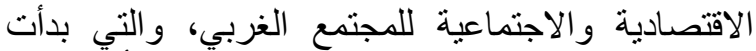
بفكر المدرسة التجارية الإنجليزية، حيث كانية الإنت أفكار تلك التك

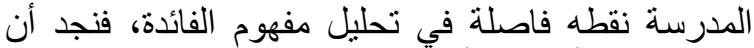
"جون لوك" أول من أوضح العلاقة بين سعر الفائدة وبين

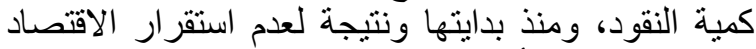

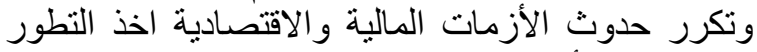

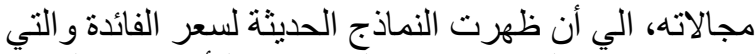
تفسر ارنباط التغير في معدلها تجاه التهاه التأثير في النشاط

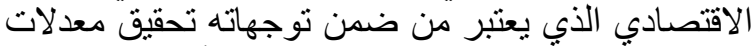

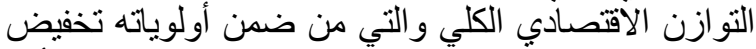

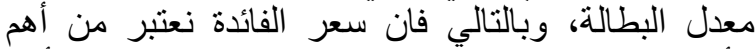

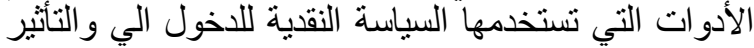

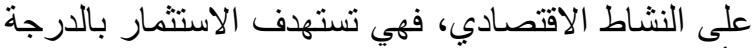

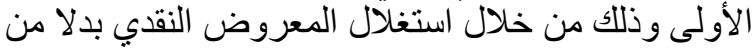

* Corresponding author: Tel. :+201144343809

E-mail address: hanyelhwary110829@gmail.com 
بتفاعل عرض أموال المدخرين و الطلب عليها من قبل

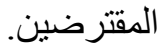

- Distribution of ) بينما عرفها كارفور في كتابه الإنيه (Wealth أن سعر الفائدة هو الذي يوازن بين الإنتاجية

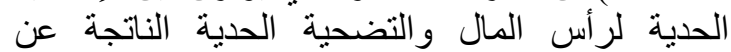

الانتظار (حسن، 2005).

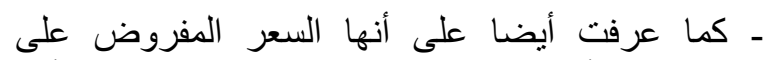

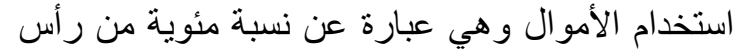

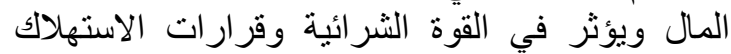

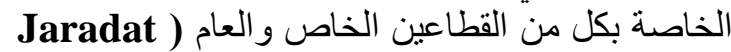

.(and Al Hosban, 2014

وبذلك يمثل سعر الفائدة المتغير الاقتصادي الذي بربط الذيط

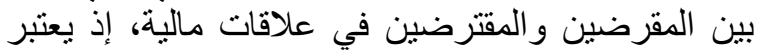

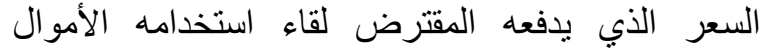
المقترضة لفترة زمنية معينة بتفق عليها (سعر الائتمان O'sullivan and ) (The price of Credit Steven, 2003 (أكن سعر الفائدة يختلف بعض الثيء الثيء عن أسعار السلع و الخدمات حيث أنه ليس سعر النئ مطلقا أنماء

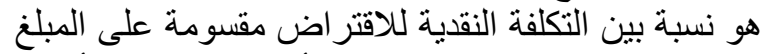

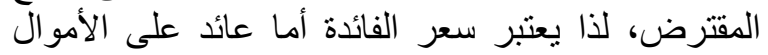
المقترضة، أو بمعني آخر فهو تكلفة لها.

سعر الفائدة كأداة من أدوات السياسة النقدية

من خلال توضيح مفهوم السياسة النقدية ومفهوم سعر

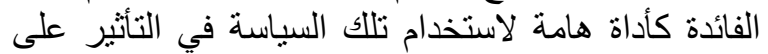
النشاط الاقتصادي وتحقيق أهدافه، يمكن توضيح استخدام

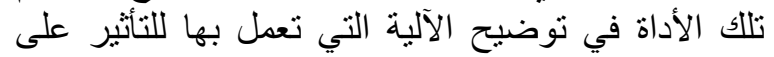

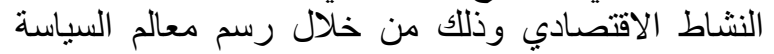

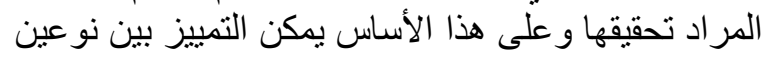
من السياسات النقدية التي يمكن تطبيقها وهي كالآتي (حاجي، 2016):

\section{السياسة النقلية الانكماثية}

وتتفذ هذه السياسة من خلال استخدام أداة سعر الفائدة عن طريق رفع سعر الفائدة، حيث تقيثة التيد حجم الايتمان وتشجع المواطنين على الادخار وبالتالي التقليل من حجم الاتح

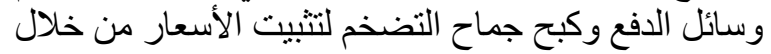

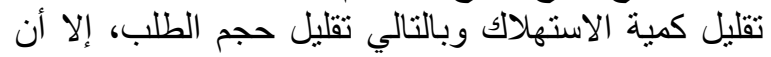

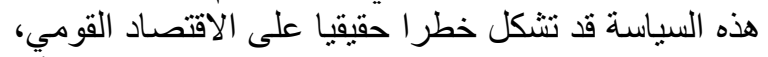

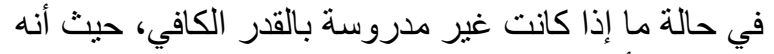

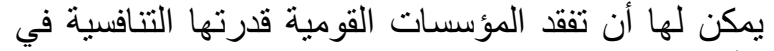

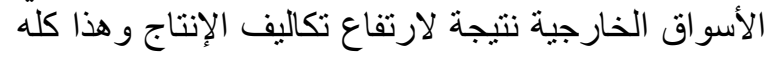

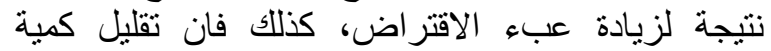

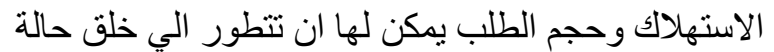
من الركود وبالتالي حدوث مشكلات الطنات اقتصادية كثيرة.
فرضية الاراسة

توجد علاقة طردية بين سعر الفائدة الحقيقي ومعدل البطالة.

منهجية الدراسة

سعيا للإجابة على الإشكالية المطروحة سلفا واختبار

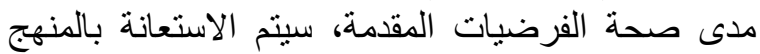

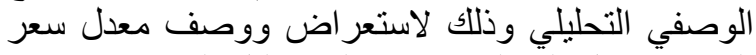

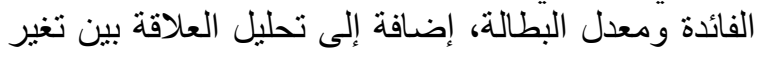

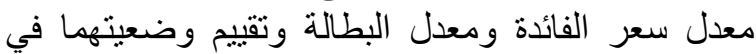

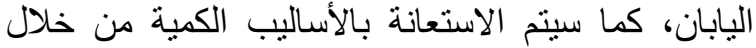
در اسة قياسية لآثار معدل سعر الفائدة ومعدل البطالئة

\section{مفهوم سعر الفائدة وعلاقته بالبطالة}

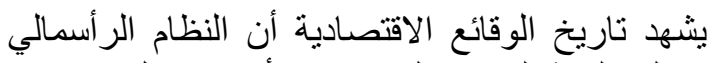

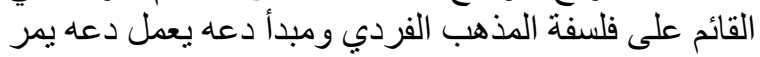

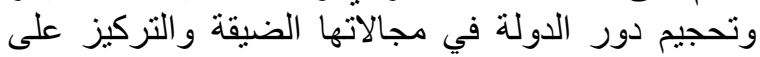

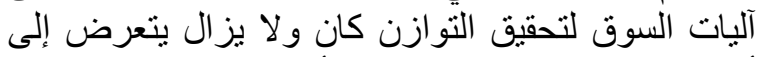

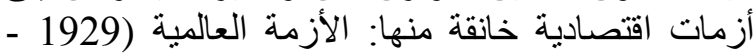

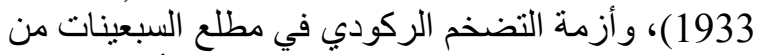
القرن العشرين وتجدد ظهورها، وكذللك الأزمة المالية اللئية المعاصرة التي اجتاحت الاقتصاد الأمريكي 2008 وبعض الاقتصاديات الر أسمالية.

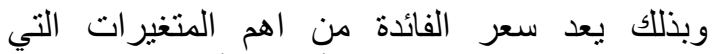

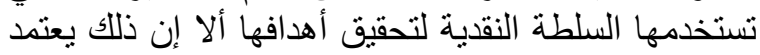

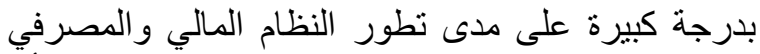

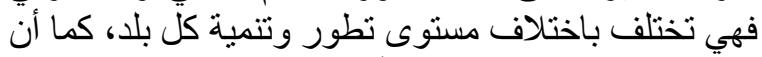

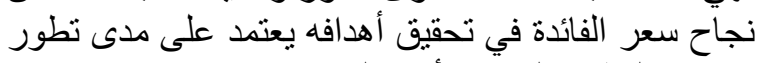

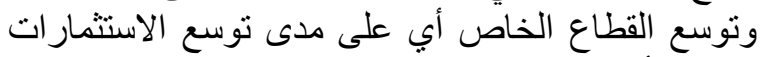
و هذا الأمر مختلف في الدول المتقدمة عنه في الدول الأني النامية أيضا.

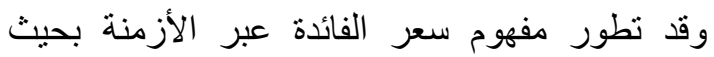

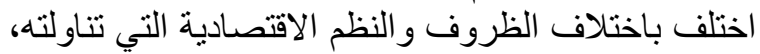

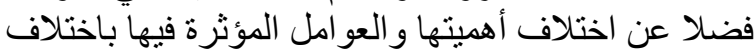

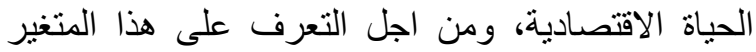
يمكن توضيح بعض المفاهيم لها كالتالي:

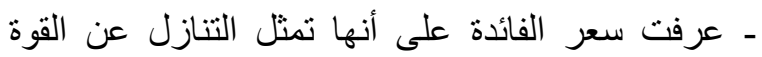

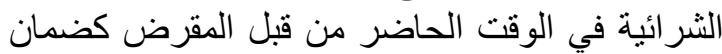

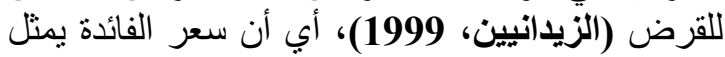

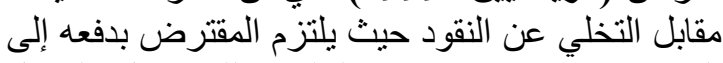
المقرض وعادة ما يكون البنلك وذللك مقابل التنازل المؤقت لله عن السيولة.

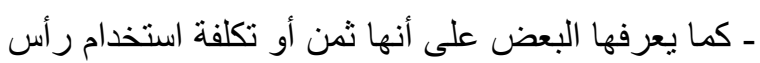
المال (محمد، عريقات، 2006)، حيث أنها أنها تتحدد قيمنها 
ذلك زيادة كمبة المعروض النقدي بنفس النسبة تجنبا

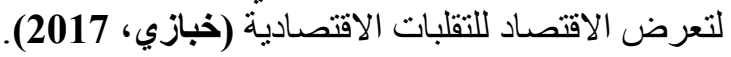

\section{Realization of full تحقيق الاستخدام الكامل} employment

حيث أن الاستخدام الكامل يعني التشغيل الكامل وزيادة

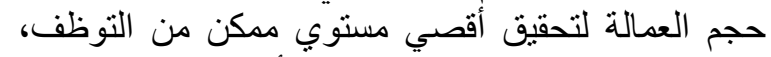

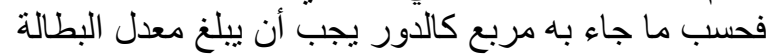

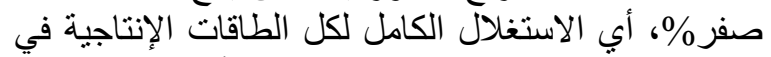
المجتمع (علّي، 2013)، ويجب الإلي ملاحظة أن سعي السياسةً

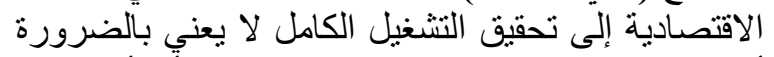

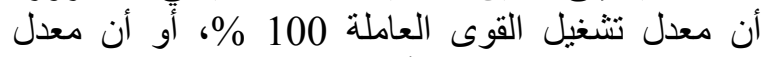

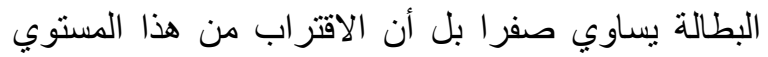
بقدر ما يمكن هو الهدف الرئيسي (كروش، 2016).

\section{Realization of تحقيق التوازن الخارجي} salable balance of payment

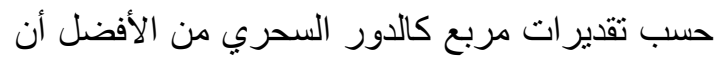

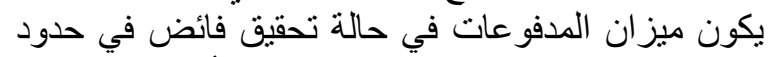

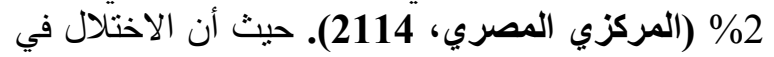
ميز ان المدفوعات ينعكس بالسلب على قيمة العيمة العملة الوطنية و أبضا تفاقم مشكلة المديونية الخارجية بلئ.

جدير بالملاحظة أن تحقيق الأهداف الأربعة السابقة

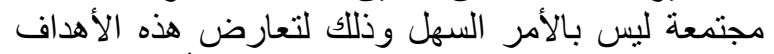

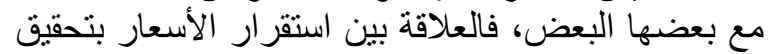

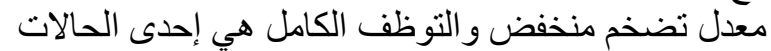

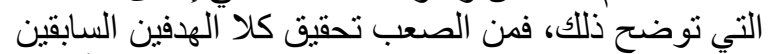

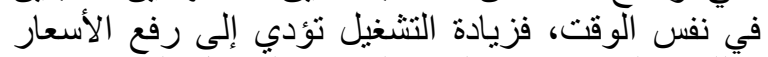

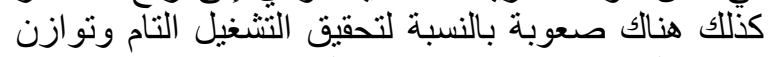

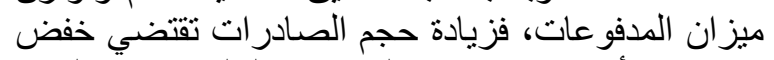
مستوى الأسعار وتحسين الجودة وبادئ الألتالي زيادة القدرة

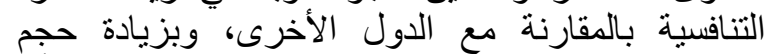

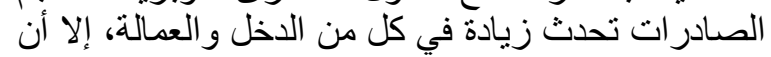

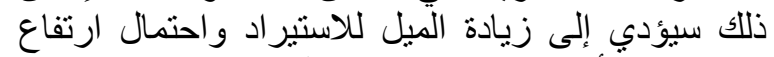

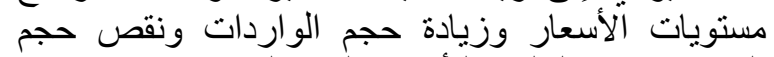

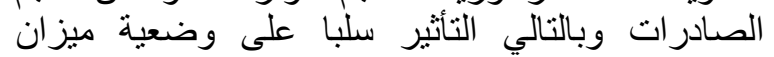

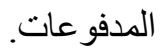

أما العلاقة بين النمو الاقتصادي واستقرار الأسعار

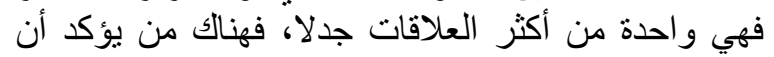

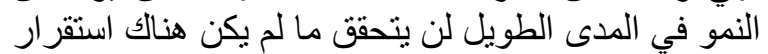

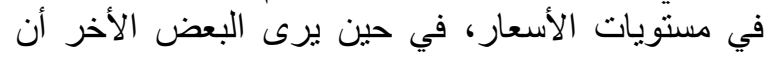

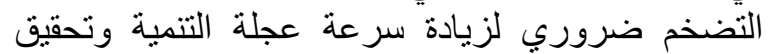

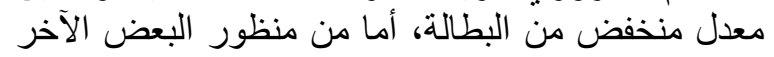

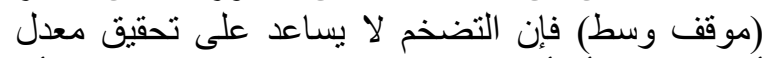

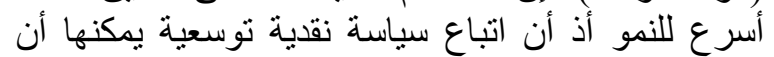

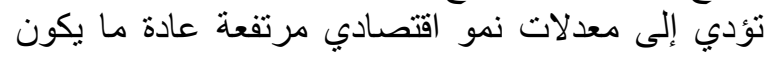
على حساب ارتفاع معدلات التضخم (ضيف، 2014).
السياسة النقدية التوسعية

وتنفذ هذه السياسة من خلال استخدام أداة سعر الفائدة

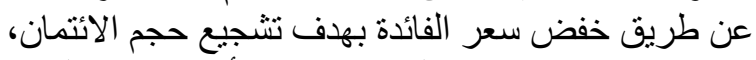

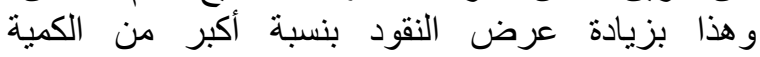

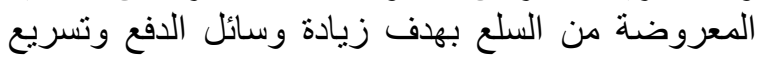

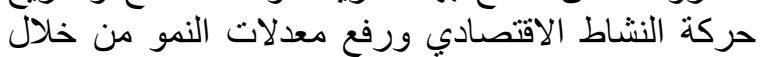

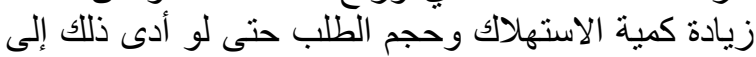

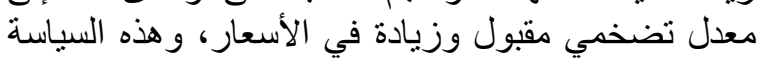
تؤدي إلى زيادة الطلب الاستثماري وتحقيق التشغيل

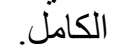

\section{سعر الفائدة كأداة لتحقيق أهداف السياسة النقدية}

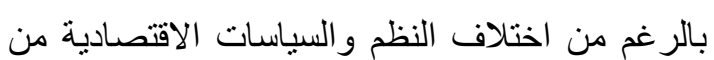

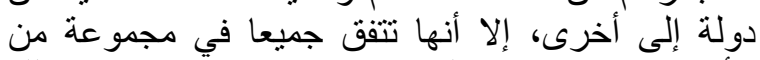

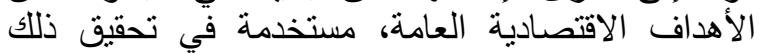

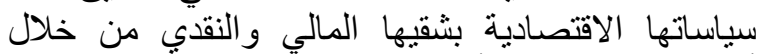

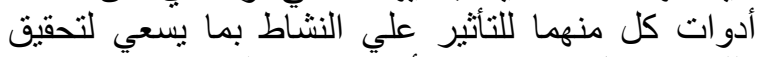

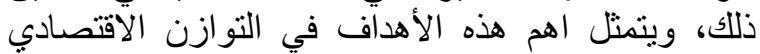

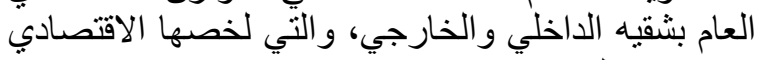

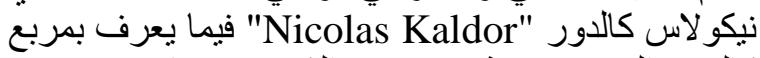

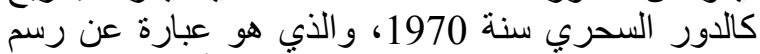
تخطيطي رباعي الرؤوس يحتوبي علي الثى الأهداف الرئيسية

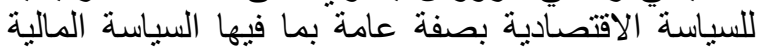

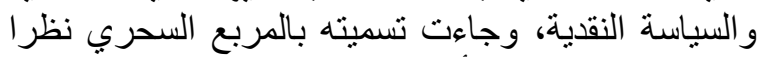

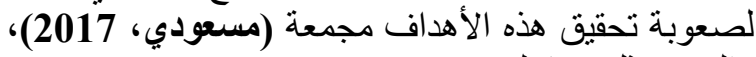
و التي تتمثل فيما يلي:

Realization of economic تحقيق النمو الاقتصادي: growth

طبقا لمربع كالدور يجب أن يبلغ معدل النمو

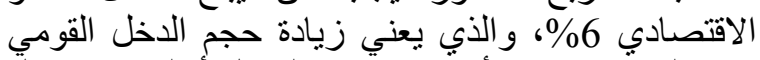

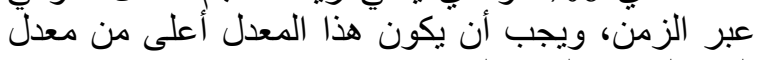
النمو السكاني لنفس الفترة (صلاح، 2016)

وهنا يقصد بمعدل النمو الحقيقي وليس الإسمي لأن

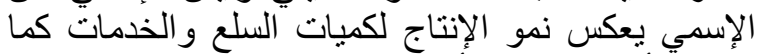

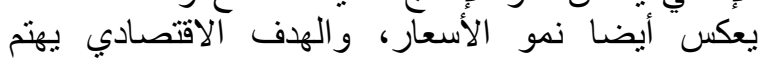

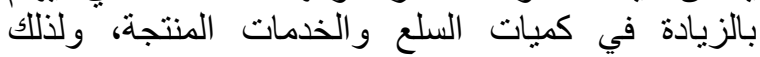
نستعمل معدل النمو الحقيقي في التحليل الاقتصـادي

(Boutaleb, 2004)

\section{Realization of تحقيق الاستقرار في الأسعار price stability}

أي التحكم في معدل التضخم و الذي يعبر عن الارتفاع

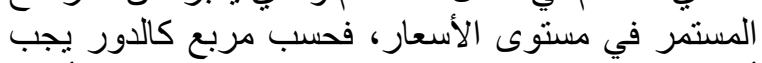

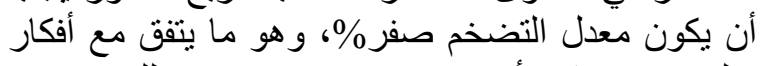

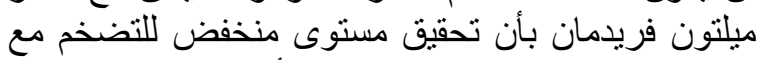

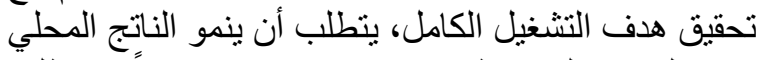

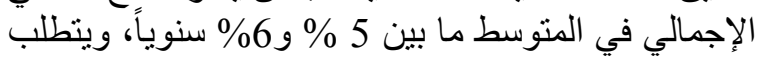


التابعة في النموذج محل الدر اسة حسب ما ذكر في البحث سابقا هي مؤشرات التوازن التهن الاقتصادي الداخلي في اليابان

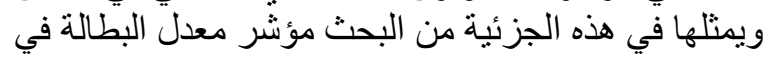

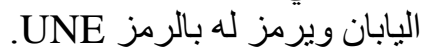

$$
\text { المتغيرات المستقلة (المفسرة) بلتيزة }
$$

هي المتغيرات التي تؤثر في المتغير التابع وليس

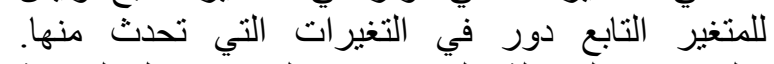

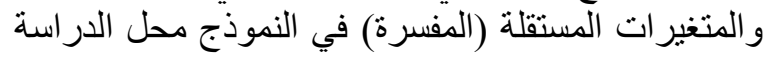

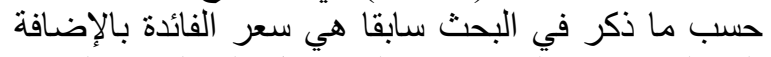

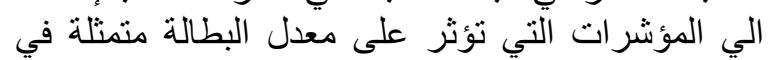

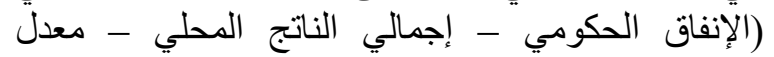

$$
\text { التضخم - عدد السكان) (لعكان) }
$$

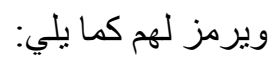

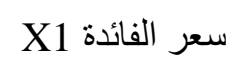$$
\text { الإنفاق العام X2 }
$$

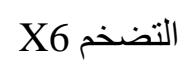

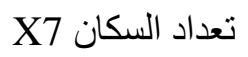

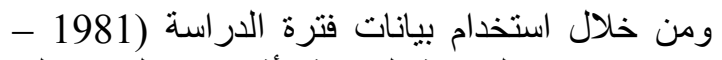
2017) م لإجر اء الدراسة القياسية لأثر سعر الفائدة على لئل

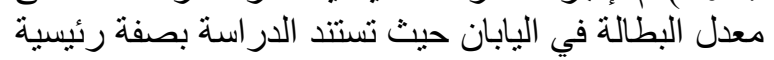

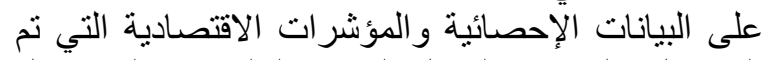

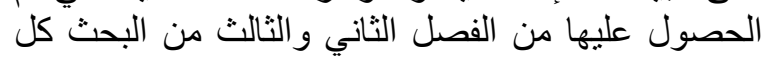
حسب مصدره و الموضحة في جدول الفحل الفي 1.
ونتيجة لصعوبة تحقيق نتائج جيدة لتلك الأهداف بسبب

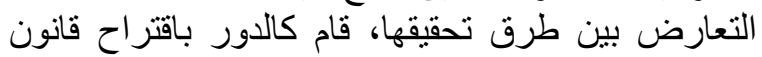

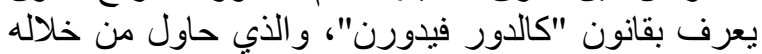

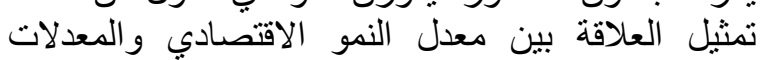

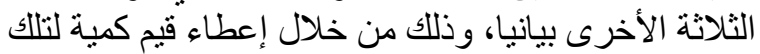
المعدلات (غرس الدين، 2008)، كما يتضح من شكل 1. تحليل أثر سعر الفائدة على معدل البطالة في اليابان

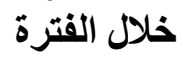
يناقش هذا الجزء من البحث التحليل القياسي لأثر سعر البان الفائدة على معدل البطالة في اليابان خلان البحل الفترة (1981-

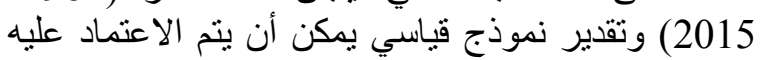

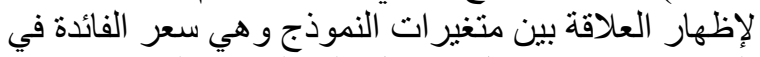
اليابان كمتغير مستقل ومعدل البطالة في الئن اليابان كمتغير

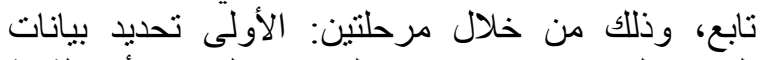

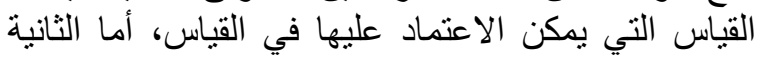

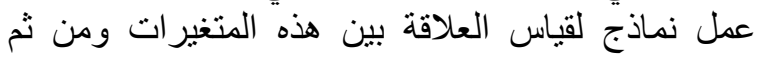
تحليل النتائج المتحصل عليها.

$$
\text { توصيف النموذج }
$$

يتم تحديد المتغيرات التي يجب إدخالها في النموذج فئج

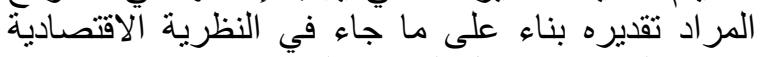
وتتقسم المتغير ات محل الدر اسة إلى نو عين رئيسيين هما:

\section{المتغيرات التابعة}

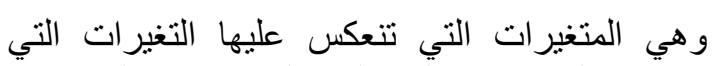
تحدث في المتغيرات المستقلة (المفسرة). والمتغيرات التيرات

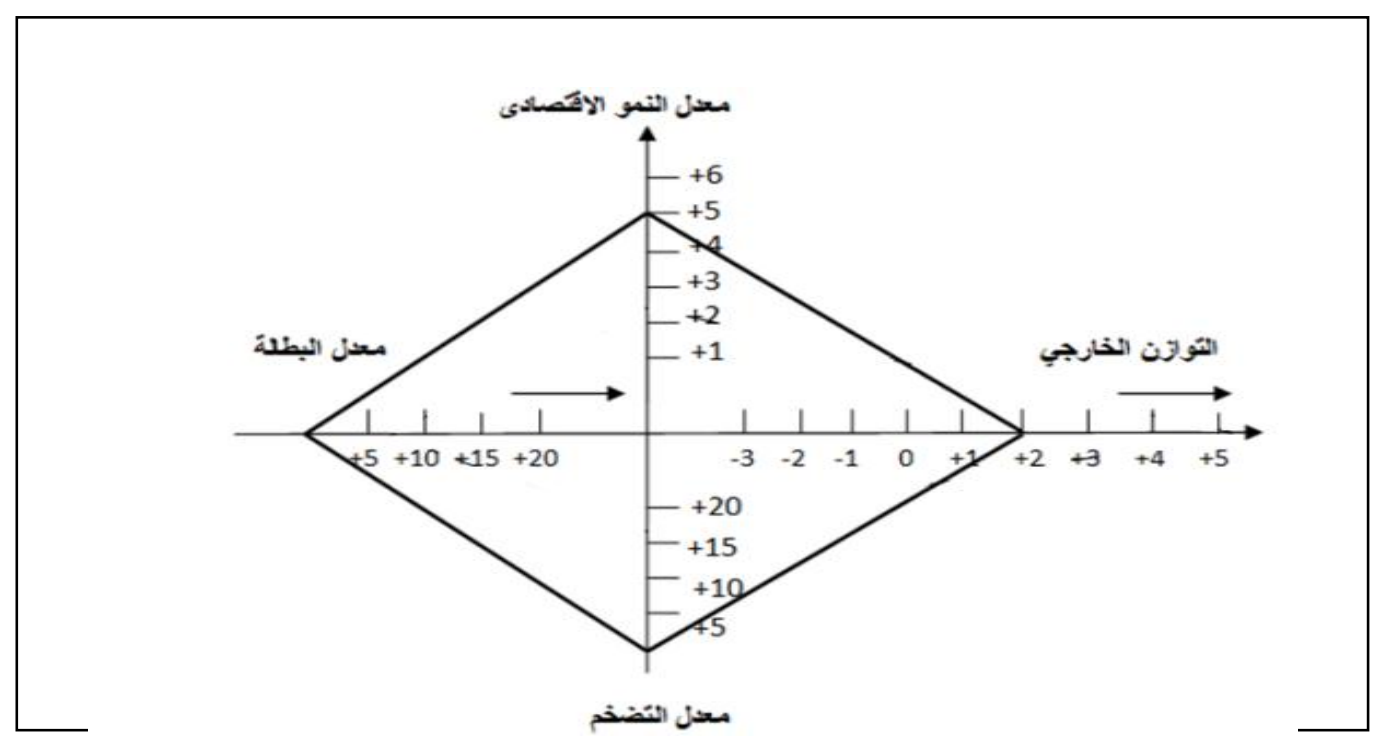

شكل 1. مربع كالدور السحري

المصدر: محمد راتول، صلاح الدين كروش، تقييم فعالية السياسة النقدية في تحقيق المربع السحري ل كالدور في الجزائر خلال الفترة (2000-

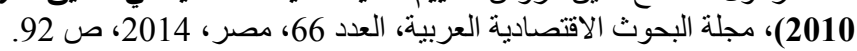


جدول 1. بيانات معدل البطالة في اليابان والمؤشرات المؤثرة فيه وسعر الفائدة خلال الفترة (1981 - 2017) م

\begin{tabular}{|c|c|c|c|c|c|c|}
\hline X5 & $\mathrm{X4}$ & X3 & $\mathrm{X} 2$ & $\mathrm{X1}$ & UNF & الرمز \\
\hline 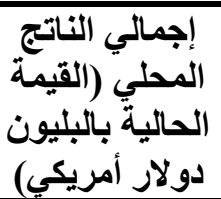 & 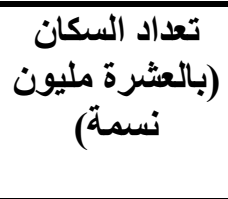 & التضخم، الأسعار & الجالجيالي الأنفاقي بالأسعار & الحقيقي (لفائدة & (القوى من إجمالة، إجمالي & $\frac{\overline{3}}{\sqrt[3]{3}}$ \\
\hline 1.22 & 11.76 & 4.91 & 1.21 & 4.80 & 2.20 & 1981 \\
\hline 1.13 & 11.84 & 2.74 & 1.13 & 5.47 & 2.40 & 1982 \\
\hline 1.24 & 11.93 & 1.90 & 1.22 & 6.12 & 2.7 & 1983 \\
\hline 1.32 & 12.00 & 2.26 & 1.29 & 5.20 & 2.70 & 1984 \\
\hline 1.40 & 12.08 & 2.03 & 1.35 & 5.27 & 2.60 & 1985 \\
\hline 2.08 & 12.15 & 0.60 & 2.00 & 4.34 & 2.80 & 1986 \\
\hline 2.53 & 12.21 & 0.13 & 2.46 & 5.37 & 2.90 & 1987 \\
\hline 3.07 & 12.26 & 0.68 & 3.01 & 3.38 & 2.50 & 1988 \\
\hline 3.05 & 12.31 & 2.27 & 3.01 & 3.11 & 2.30 & 1989 \\
\hline 3.13 & 12.35 & 3.08 & 3.11 & 4.15 & 2.10 & 1990 \\
\hline 3.58 & 12.39 & 3.25 & 3.53 & 4.47 & 2.10 & 1991 \\
\hline 3.91 & 12.42 & 1.76 & 3.83 & 4.41 & 2.20 & 1992 \\
\hline 4.45 & 12.45 & 1.24 & 4.36 & 4.27 & 2.50 & 1993 \\
\hline 4.91 & 12.50 & 0.70 & 4.81 & 3.86 & 2.90 & 1994 \\
\hline 5.45 & 12.54 & -0.13 & 5.38 & 4.06 & 3.20 & 1995 \\
\hline 4.83 & 12.58 & 0.14 & 4.81 & 3.17 & 3.40 & 1996 \\
\hline 4.41 & 12.61 & 1.75 & 4.37 & 1.94 & 3.40 & 1997 \\
\hline 4.03 & 12.64 & 0.66 & 3.96 & 2.37 & 4.10 & 1998 \\
\hline 4.56 & 12.66 & -0.34 & 4.49 & 3.52 & 4.70 & 1999 \\
\hline 4.89 & 12.68 & -0.68 & 4.82 & 3.50 & 4.70 & 2000 \\
\hline 4.30 & 12.71 & -0.74 & 4.28 & 3.11 & 5.00 & 2001 \\
\hline 4.12 & 12.74 & -0.92 & 4.06 & 3.37 & 5.40 & 2002 \\
\hline 4.45 & 12.77 & -0.26 & 4.37 & 3.50 & 5.30 & 2003 \\
\hline 4.82 & 12.78 & -0.01 & 4.72 & 2.90 & 4.70 & 2004 \\
\hline 4.76 & 12.78 & -0.28 & 4.68 & 2.74 & 4.40 & 2005 \\
\hline 4.53 & 12.79 & 0.25 & 4.47 & 2.57 & 4.10 & 2006 \\
\hline 4.52 & 12.80 & 0.06 & 4.43 & 2.63 & 3.90 & 2007 \\
\hline 5.04 & 12.81 & 1.38 & 5.02 & 2.92 & 4.00 & 2008 \\
\hline 5.23 & 12.80 & -1.35 & 5.20 & 2.35 & 5.10 & 2009 \\
\hline 5.70 & 12.81 & -0.72 & 5.62 & 3.56 & 5.10 & 2010 \\
\hline 6.16 & 12.78 & -0.27 & 6.19 & 3.23 & 4.52 & 2011 \\
\hline 6.20 & 12.76 & -0.05 & 6.30 & 2.19 & 4.30 & 2012 \\
\hline 5.16 & 12.74 & 0.35 & 5.28 & 1.64 & 4.00 & 2013 \\
\hline 4.85 & 12.73 & 2.76 & 4.97 & -0.52 & 3.60 & 2014 \\
\hline 4.39 & 12.71 & 0.79 & 4.41 & -0.98 & 3.40 & 2015 \\
\hline 4.92 & 12.70 & -0.12 & 4.87 & 0.77 & 3.10 & 2016 \\
\hline 4.87 & 12.68 & 0.47 & 4.82 & 1.23 & 2.80 & 2017 \\
\hline
\end{tabular}

http://data.albankaldawli.org/country/japan

http://ar.knoema.com/atlas/ Country/japan 


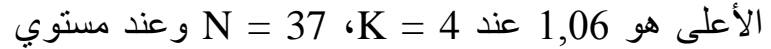
معنوية 1\% و والحد الأدنى هو 1,51) (سليمان وآخرون،

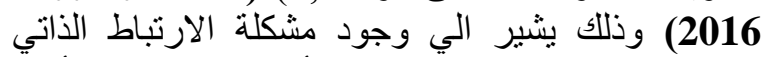
Autocorrelation للقيمة الجدولية.

وبعد أن قام الباحث بتقدير نموذج الانحدار للمتغير ات البهات

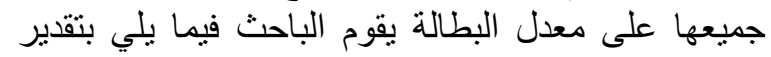

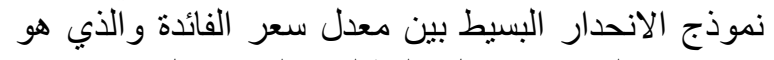

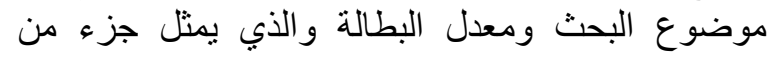
التوازن الداخلي وذللك على النحو التالي.

تقدير النموذج بين سعر الفائدة ومعدل البطالة في اليابان خلال الفترة (1981-2017) باستخدام نموذج سئج الانحدار البسيط البان

$\mathrm{UNE}=2.88-0.16 \mathrm{X} 1$

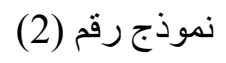

$(10.96)^{* * *}(2.24)^{*}$

$\mathrm{R}^{2}=0.354 \quad \mathrm{R}^{2}=0.125 \quad \mathrm{~F}_{\mathrm{c}}=5.003^{*} \quad \mathrm{D} \cdot \mathrm{W}=.199$

بيضح من نتائج النموذج رقم (2) وجود علاقة عكسية

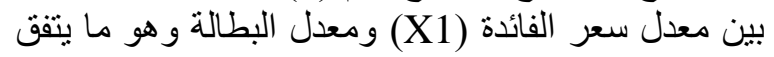

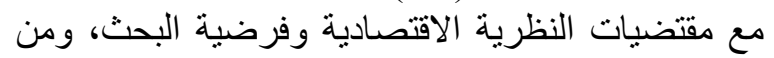

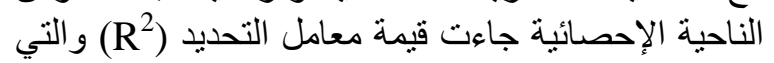

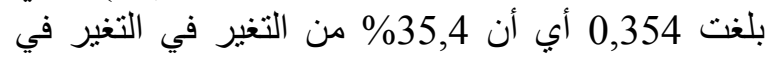

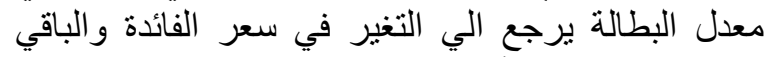

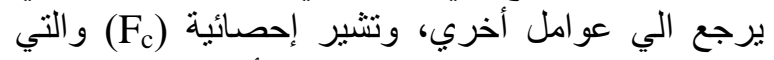

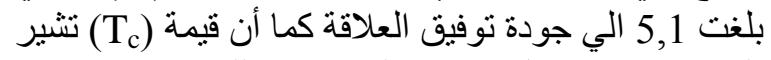

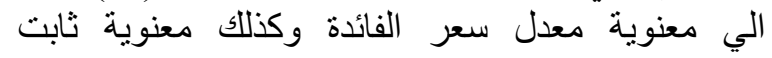

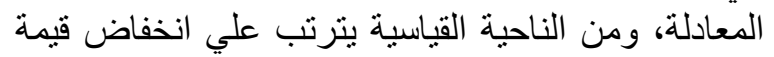

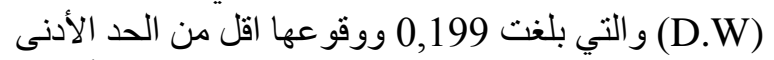
الي وجود مشكلة الارتباط الذاتي بين الأخطاء

.Autocorrelation

\section{النتائج}

1- حققت اليابان اقل منوسط في سعر الفائدة الحقيقي في

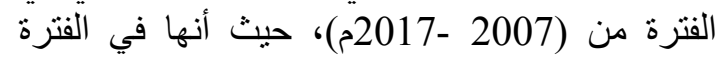

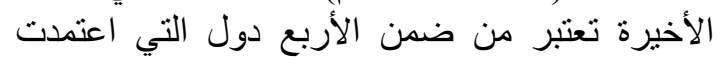
سعر الفائدة السالبة في سياستها النقدية.

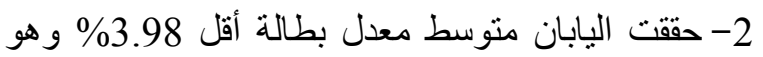

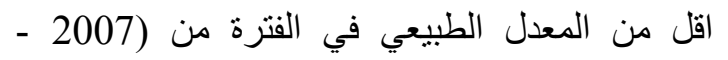
(2017

3- أسفرت نتائج التحليل القياسي للبيانات أن سعر الفائدة في اليابان يتناسب طردي مع معدل البطالة.
ويمكن توضيح المؤشر ات بيانيا من خلال شكل 2.

تقدير النموذج القياسي

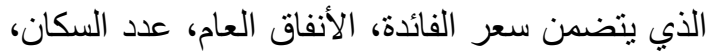

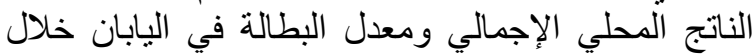
الفترة (1981 - 2017) في الشكل الخطي حيث أسفرت النيان

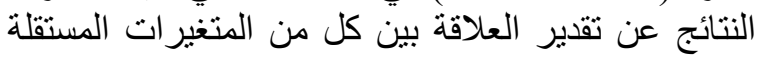

و المنغير التابع عما يلي:

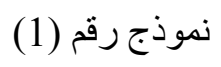

$\mathrm{UNE}=-47.55+0.237 \mathrm{X} 1+2.61 \mathrm{X} 2+4.04 \mathrm{X} 7-0.003 \mathrm{X} 9$ $(5.86)^{* *}(3.30)^{* *}(1.77)^{\mathrm{NS}}(5.98)^{* *}(1.17)^{*}$

$\mathrm{R}^{2}=0.848 \quad \mathrm{R}^{2}=0.718 \quad \mathrm{~F}_{\mathrm{c}}=20.419^{* *} \mathrm{D} . \mathrm{W}=.586$

$$
\text { يتضح من نتائج النموذج رقم (1) ما يلي: }
$$

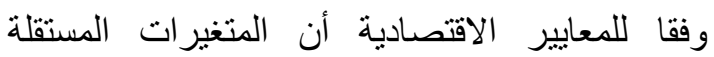

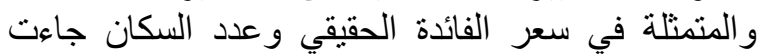

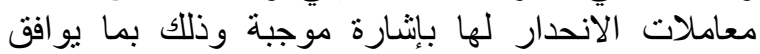

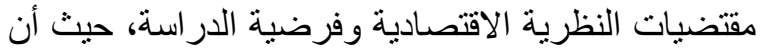

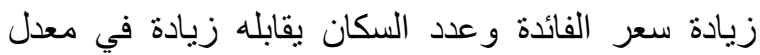

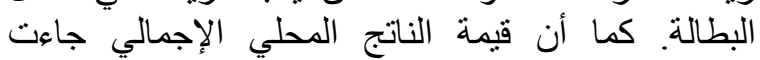

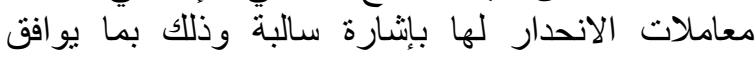

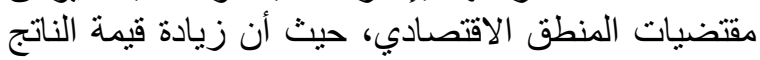

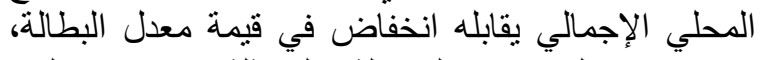

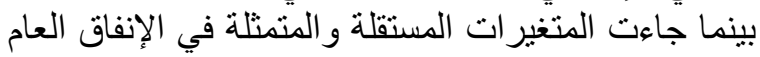

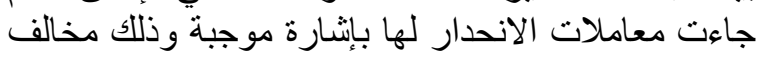

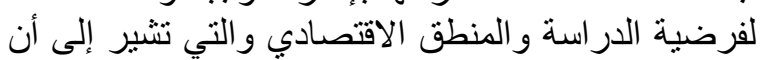

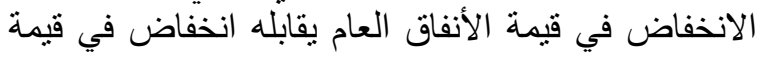
الاستثمار وبالتّالي زيادة في معدل البطان البالة.

وفقا للمعايير الإحصائية بلغت قيمة معامل التحديد

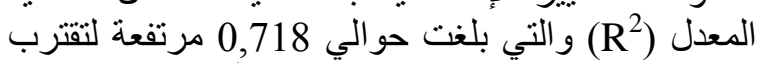
من الواحد الصحيح وهو ما يشير إلي أن هذه المتغير التيرات

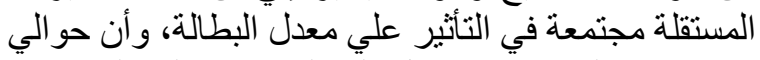

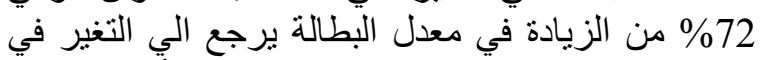

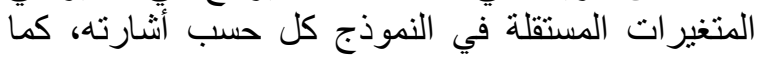

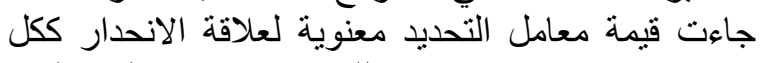

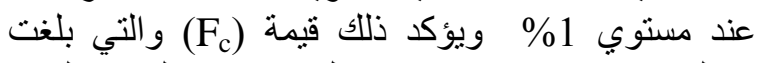

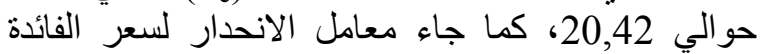

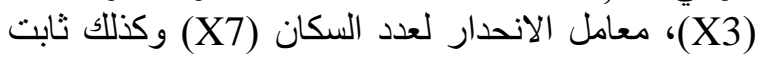

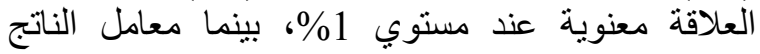

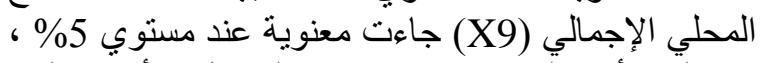
كما لم تتأكد المعنوية الإحصائية لمعامل الأنفاق لئ العام

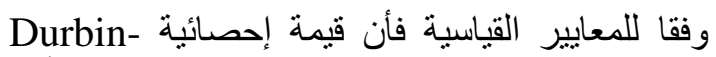
(D.W)Watson اقل من الحد الأدنى للقيمة الجدولية الإحصائية (الحد 


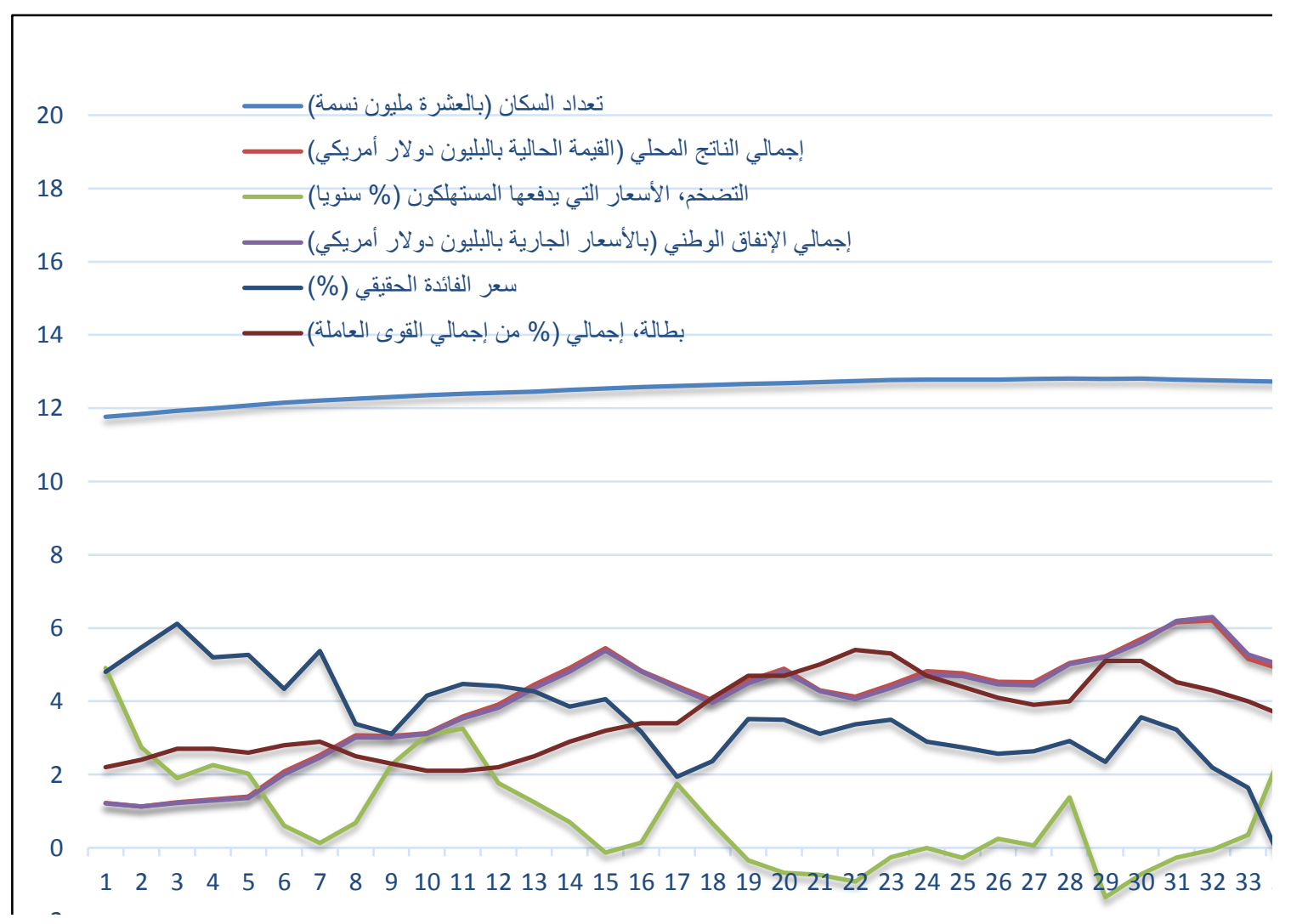

شكل 2. مؤشرات الإنفاق الحكومي، إجمالي الناتج المحلي، معدل التضخم، عدد السكان ومعدل البطالة

المصدر: من أعداد الباحث اعتمادا على بيانات جدول 1.

حسن، جمال عبد المجيد عبد الصمد (2005). دور سعر الفائدة في استقرار سعر الصرف في مصر ، الصدة رسالة ماجستير قسم الاقتصاد كلية التجارة جامعة عين رلفرال

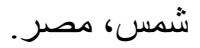
خبازي، فاطمة الزهر اء (2017). اقتصاد المعرفة ودوره

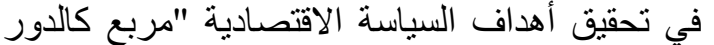

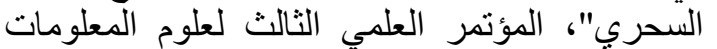

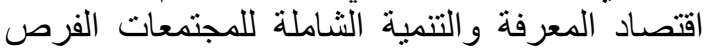
و التحديات، 10 - 11 أكتوبر بجامعة بني سويف، لتصني

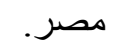
الزيدانيين، جميل سالم (1999). أساسيات في الجهاز المالي، ط 1، دار و وائل للطباعة و النشر، عمان.

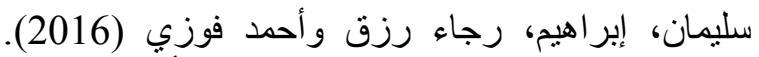
مقدمة في الاقتصاد القياسي، الطبعة الأولي، المكتبة الأكاديميةً، القاهرة. صلاح، محمد (2016). أهداف السياسة الاقتصادية الكلية

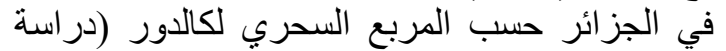

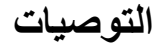

1- ضرورة تفعيل دور السياسة النقدية واستخدام أدواتها

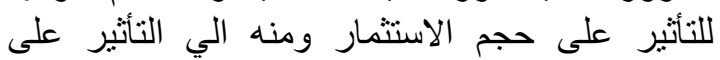
معدلات النمو و البطالة.

2- خفض معدلات سعر الفائدة كأداة من أدوات السياسة

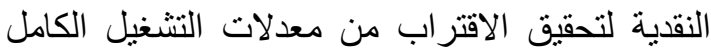
وخف معدلات البطالة.

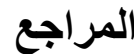

البنك المركزي المصري (2014). المعهذ المصرف العربي، مفاهيم مالية، العدد 12، القاهرة، مصر.

حاجي، سمية (2016). دور السياسة النقدية في معالجة اختلال ميزان المدفوعات حالة الجزائر (19902014)، رسالة دكتوراة، كلية العلوم الاقتصادية، جامعة محمد خيضر بسكرة، الجزائر. 


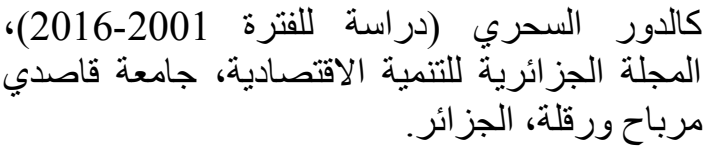

http://ar.knoema.com/atlas/Country/japan

http://data.albankaldawli.org/country/japan

Jaradat, M.A. and S.A. Al Hosban (2014). Relationship and Causality Between Interest Rate and Inflation Rate case of Jordan, Interdisciplinary J. Contemp. Res. Business, $6: 4$.

Kouider Boutaleb, The policy of the effectiveness of economic policies in P.V.D. The case of Algeria international conference on economic policies in Algeria, realities and perspectives, Tlemcen, 2004.

O'sullivan, A. and M. Steven (2003). Macroeconomics, $6^{\text {th }}$ Ed, New Jersey.

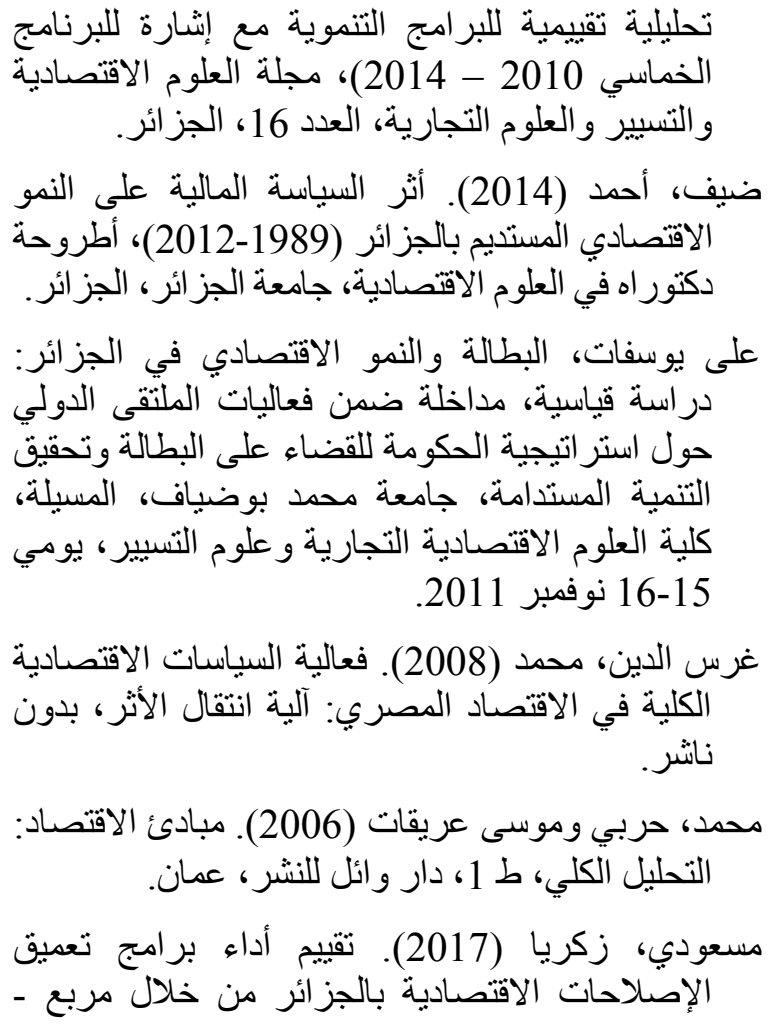

\section{IMPACT OF LOW INTEREST RATES ON JAPAN'S UNEMPLOYMENT RATE}

\section{Hany M.S. Hawary ${ }^{1}$ and A.F. Hamed ${ }^{2}$}

1. Polit. and Econ. Sci. Studies and Res. Dept., Asian Studies and Res. Inst., Zagazig Univ., Egypt

2. Agric. Econ., Fac. Agric., Zagazig Univ., Egypt

ABSTRACT: The interest rate is an important tool of monetary policy. Japan has changed the concept of using the interest rate as a tool to achieve low rates of unemployment through the use of the low interest rate, and it has even reached what is known as the "zero interest rate". Consequently, the research problem revolves around identifying the reality of the interest rate in Japan, and identifying its contribution to reducing the unemployment rate in Japan by analyzing the data for the study period (1981-2017). The most important results indicated that Japan achieved the lowest average in the real interest rate in the period from (2007-2017), as in the recent period it is among the four countries that adopted the negative interest rate in its monetary policy, as economic statistics indicate that Japan, Sweden, Denmark and Switzerland Negative interest rates are based on $-0.1 \%,-0.3 \%,-0.7 \%$ and $0.8 \%$, respectively. The research recommends, the necessity of using a monetary policy that seeks to reduce the interest rate to push the investment wheel and from it to reduce the unemployment rate.

Key words: Japan, interest rate, unemployment rate, monetary policy. 